Cahiers de recherches médiévales

\title{
Aspects du vocabulaire politique et social chez Oresme et Christine de Pizan
}

Vers une nouvelle conception de l'État et de la société

\section{Charles Brucker}

\section{(2) OpenEdition \\ Journals}

Édition électronique

URL : https://journals.openedition.org/crm/408

DOI : $10.4000 / \mathrm{crm} .408$

ISSN : 1955-2424

\section{Éditeur}

Honoré Champion

Édition imprimée

Date de publication : 15 janvier 2001

Pagination : 227-249

ISSN : $1272-9752$

\section{Référence électronique}

Charles Brucker, "Aspects du vocabulaire politique et social chez Oresme et Christine de Pizan », Cahiers de recherches médiévales [En ligne], 8 | 2001, mis en ligne le 13 mars 2008, consulté le 15 décembre 2022. URL : http://journals.openedition.org/crm/408; DOI : https://doi.org/10.4000/crm 408

Ce document a été généré automatiquement le 15 décembre 2022.

Tous droits réservés 


\title{
Aspects du vocabulaire politique et social chez Oresme et Christine de Pizan
}

Vers une nouvelle conception de l'État et de la société

\author{
Charles Brucker
}

Pour Liliane Dulac, en amical hommage.

1 On sait combien les écrivains ayant gravité autour de Charles V ont été influencés par son envergure intellectuelle, culturelle et politique. C'est en effet à partir du règne de ce dernier que la littérature est directement impliquée dans la construction de la société et dans la vision politique du souverain. Jamais encore un roi ne s'est vu dédicacer autant d'ouvrages de nature, d'ailleurs, très diverse. Mais, parmi eux, ce sont certainement ceux d'Oresme et de Christine qui sont le plus marqués par la personnalité et les idées politiques de Charles V. Toutefois, l'orientation respective de ces deux auteurs est sensiblement différente, dans la mesure où Oresme est une sorte de technicien de la vulgarisation, en langue française, des idées politiques et sociales d'Aristote, tandis que Christine analyse la société, mais une société centrée autour d'un roi, Charles $\mathrm{V}$, et celui-ci, à chaque allusion, fait l'objet d'un éloge sans réserve ${ }^{1}$. Cette différence et cette complémentarité des deux auteurs se manifestent tout particulièrement à travers l'emploi qu'ils font d'un certain nombre de termes relevant de la politique et de la vie politique.

\section{I.- Gouvernement et morale}

2 Le XIV ${ }^{e}$ siècle est l'époque où, en langue vernaculaire, une réflexion générale est portée sur l'organisation de la cité et sur les divers modes de gouvernement, et ce très souvent à partir de l'examen de sources ou de documents anciens, gréco-latins, et tout 
particulièrement dans le cadre de la diffusion des philosophies et des conceptions politiques antiques.

\section{Policie et la notion de gouvernement en général}

\section{$1^{\circ}$ Chez Oresme}

3 C'est aussi l'époque où des termes nouveaux sont créés. Le terme de policie ${ }^{2}$ apparaît bien dans l'œuvre d'Oresme, notamment dans les Politiques d'Aristote ${ }^{3}$.

\section{a) « Organisation politique »}

Oresme donne de la policie une définition très générale :

Policie est l'ordenance du gouvernement de toute la communité ou multitude civile. Et policie est l'ordre des princeys ou offices publiques. Et est dit de polis en grec, qu'est multitude ou citét ${ }^{4}$ (Politiques ${ }^{5}$. Glose ${ }^{6}$, p. 373a).

5 En fait, cette définition est double. D'une part, le terme recouvre les concepts d'organisme et d'organisation politiques de la communauté des citoyens, qui, étymologiquement et sémantiquement, se trouve à la base de cet aspect de la définition, aspect externe. D'autre part, un côté interne de la définition réside dans la présentation du contenu du concept évoqué : «Policie est l'ordre des princeys... ». En somme, la policie est aussi un terme collectif recouvrant l'ensemble des charges administratives de la cité ${ }^{\prime}$; donc indirectement intervient la notion de citoyens, ici encore. On constatera que, dans l'une et l'autre partie de la définition, l'idée de totalité est soulignée par toute et publiques.

Aussi n'est-il pas étonnant de voir policie prendre en charge le sémème " État » :

[...] car la fin a quoy resgardent ceulz qui ordennent la policie et la communité et qui sont aussi comme architecton, c'est delectacion, pour ce que nus dison une chose estre simplement bonne et l'autre simplement mauvaise selon ce. (Éthiques. Texte, t. II, p. 400).

7 Le terme de communité oriente nettement la valeur de policie. D'ailleurs, dans ce sens, policie est concurrencé par chose publique :

Et la chose publique selon ce que dit Plautarchus est aussi comme un corps humain.

(Politiques. Glose, p. 291a).

\section{b) « Administration en tant qu'activité publique »}

8 Plus rarement, le terme de policie désigne l'administration, l'action d'administrer. C'est notamment le cas lorsque le terme est suivi d'un complément déterminatif représentant une collectivité :

Ne le gouverneur ${ }^{8}$ de la policie du païs ou de la cité ne conseille pas se il fera ou querra bonne paix en son pueple. (Éthiques. Texte ${ }^{9}$, t. I, p. 190).

Ici, la valeur de policie est orientée par celle de païs et de cité. D'autant que le contexte est tel que le verbe gouverner implique un objet qui demande une maîtrise, une conduite, une gestion. De fait, dans les lignes qui précèdent et qui appartiennent encore au chapitre VII, il est question à plusieurs reprises de gouverner une naif; en particulier, la décision et la réflexion sont de nature différente quand il s'agit de conduire un bateau ou quand il s'agit de lutter, exercice de gymnastique, parce que, dans le second cas, l'élément intellectuel est moins présent : 
Et avecques ce de telles choses il est ainsi que il convient plus conseillier es unes que es autres; si comme en art de gouverner une naif ou tout une navie, il convient plus conseillier que en art de luitier, de tant comme il a moins de certaineté et plus de doubte en art de gouverner une naif que en l'autre. (Éthiques. Texte, t. II, p. 190).

Bien plus, il arrive que policie soit franchement synonyme de gubernacion :

Et pour ce, en gubernacion ou policie juste et droituriere, nous ne laisson ou souffron quelconquez personne ait princei, dominacion ou seigneurie selon homme, mais selon raison et selon la loy mise. (Éthiques. Texte, p. 301).

\section{$2^{\circ}$ Chez Christine de Pizan}

10 Tout comme chez Oresme, le terme de gouvernement est associé au contexte dans lequel figure policie :

Mais neantmoins voit on à la foiz des plus grans clers, si que dit la rusé, et il est vray, n'est pas les plus sages, c'est assavoir les plus prudens en fait de gouvernement et de policie, mesmement en parolle, combien que clergié l'aprengne et le demonstre. (Paix ${ }^{10}$, p. 69).

11 L'art de gouverner et l'organisation ou l'administration de la cité se rejoignent dans la sagesse et la prudence, mais il s'agit d'une sagesse et d'une prudence pratique, d'une clairvoyance qui s'inscrit dans une compétence, pratique, elle aussi. Or, Christine insiste sur le fait que ce ne sont pas les clers qui remportent la palme dans ce domaine, et ce même pas au plan du discours, de l'art de s'exprimer. L'auteur émet une critique implicite à l'encontre de la rhétorique, qui, manifestement, n'est pas d'une efficacité suffisante dans l'exercice d'une charge publique.

12 Ce qui est remarquable chez Christine dans l'emploi du terme, c'est l'abondance de qualifications élogieuses concernant ou entourant la notion portée par policie; ces qualifications peuvent d'ailleurs impliquer des exigences de la part de Christine, puisqu'elles indiquent en somme quel serait l'idéal du gouvernement d'un État ou d'une Cité :

Nonpourtant c'est bien droit que chascune porte tel abit et estat qu'apertient a son mary et a elle ; mais se elle est bourgoise que elle le porte tel que une damoiselle, et la damoiselle come une dame, et ainsi de degré en degré en montant, sans faille c'est chose hors ordre de bonne policie, en laquelle, se elle est bien ordonnee, en quel que païs que ce soit, toutes choses doivent estre limitees. (Trois Vertus ${ }^{11}$, III 2, p. 178 lignes 25-31).

13 La notion de "gouvernement, administration » est définie, ici du moins, de manière restrictive : d'une part, c'est l'ordre de bonne policie qui détermine le comportement et la place de la femme dans la société ; d'autre part, cet ordre ${ }^{12}$ s'inscrit lui-même dans un principe plus général, celui de la modération (limitees).

Quelquefois, le terme est simplement placé dans un réseau contextuel comportant certaines des qualités requises pour que le gouvernement soit efficace :

Item, pour bien gouverner le fait de la policie de son royaume, voult avoir notables clercs legistes expers afin que par leurs consaulx selon ordre de droit peust toutes choses bien disposer dont lui an ensuivi que tant qu'il regna, par tenir les manieres que ordre royalle bien ordonné requiert, tint son royaume en grant magnificence et croissement de felicité de mieux en mieulx [...] (Charles $V^{13}$, t. I, p. 123).

15 La conduite des affaires publiques et notamment de l'organisation politique du royaume implique, de la part de l'entourage immédiat du roi, une spécialisation ${ }^{14}$ : les vocables notables-legistes-expers mettent en valeur cette idée. Dans le réseau contextuel entrent aussi les expressions ordre ${ }^{15}$ de droit-ordre royalle-bien ordonné se situent dans un 
champ sémantique voisin, celui d'une organisation rationnelle de la société, autre thème représentatif du XIV ${ }^{e}$ siècle $^{16}$. En fait, policie glisse ici vers la notion de " État ».

Dans ce sens plus général, policie peut alors être concurrencé par chose publique, désignant l'État, tout comme chez Oresme :

[À propos de la comparaison de l'État avec le corps humain]

Tout ainsi, ce dit il, est de la chose publique : Oste les baboureux et ceulx qui font

les mestier, elle ne se pourra soustenir... (Policie ${ }^{17}$, p. 195).

\section{Le princey et l'office dans la société}

L'office ou princey sera la cheville ouvrière du nouvel édifice politique et social qui est en train de se construire dans l'esprit des théoriciens et des écrivains de l'époque de Charles V.

\section{a) Chez Oresme}

18 Le terme d'office oscille constamment entre le devoir au sens d'obligation morale en général et l'obligation particulière qu'exige ou que comporte une charge ou une dignité au sein de l'organisme social et politique qu'est la cité.

19 Le sens de "devoir " semble subordonné à celui de "charge ». Toutefois, dans les Éthiques d'Aristote traduit en français par Oresme, les deux notions semblent se conditionner réciproquement. Ainsi, à propos de la question « a savoir mon, se le juge doit condempner celui que il scet certainement estre innocent ou cas que il seroit prouvé par tesmoins en forme de droit que il est coulpable » (Éthiques. Commentaire, t. II, p.316). Après l'évocation de certains raisonnements émis sur ce point par les théologiens et les juristes, Oresme ${ }^{18}$ en arrive à présenter une dernière opinion de certains théologiens :

Secondement, il appartient principalment au juge garder et tenir a l'angle tous tels droiz; car c'est son office. (Ibid.)

Donc, la fonction de juge implique, entre autres, le devoir bien précis de veiller au respect des lois. D'ailleurs, de ce point de vue, il convient de rappeler la définition qu'Oresme donne du Livre des Éthiques dans son prologue aux Éthiques: «Le Livre des Éthiques, c'est livre de bonnes meurs, livre de vertus ouquel il enseigne, selon raison naturel, bien faire et estre beneuré en ce monde. " (Éthiques. Texte, t. I, p. 97). Or, les bonnes moeurs, les vertus sont inséparables de la notion de devoir d'une manière générale, puisque, en fin de compte, le devoir en tant qu'obligation conditionne l'existence même de chaque vertu.

21 Dans l'œuvre d'Oresme, notamment dans les Politiques, si un terme fait singulièrement concurrence à office, c'est celui de princé/princey ${ }^{19}$ :

Et celz princeys sunt ceuls qui sunt ordenés pour l'oportunité des guerres ou des batailles. (Politiques. Texte, p. 273a)

Et tous ces expediens quant a cest princey ou office ont lieu mesmement es .II. guerres ou manieres de policie (Politiques. Glose, p. 273a).

Dans les deux cas, les circonstances de la vie politique dans lesquelles s'inscrivent les offices sont clairement indiquées par Oresme, sinon par Aristote lui-même. 


\section{b) Chez Christine} tourbillon de l'instabilité des changements et bouleversements de la condition humaine. Etant donné que, souvent, Christine fait entrer dans la danse des vicissitudes le gain et la richesse pour aussitôt en montrer la fragilité et l'instabilité, office est plus d'une fois associé à benefice :

$-[\ldots]$

Mais, qui par la peut ens passer [v. 2173]

Trop grant avoir peut amasser,

Car puisque Eur lui aura mis,

A Richece sera amis,

Si aura ou chastel office

Bon et bel ou grant benefice,

s'il est clerc, quelque peu qu'il sache,

Puisque Richece ens le sache,

Se cellui qui fait l'avant garde

De ma dame ne l'en deboute...]. (Mutacion ${ }^{21}$, t. I, p. 83).

dans le contexte du passage évoqué, ce n'est pas la cité, mais le château qui est le centre des activités qui englobent l'office. Le terme d'office, aussi bien que celui de benefice, se trouvent singulièrement dévalorisés : le clerc qui obtient l'un ou l'autre, peut, en somme, être parfaitement ignorant ; ce qui veut dire que ce n'est pas le mérite qui justifie l'obtention d'une charge ou d'un titre; Fortune avec son pouvoir destructeur est toujours aux aguets pour retirer ce qu'elle a donné (debouté).

En face de cette situation, Christine évoque la rigueur du système politique et social des Anciens :

Et pour ce pleust à Dieu que, ainsi que autre fois ay dit ailleurs sur ceste matiere, que ordre fust par tout de tenir en telz cas les manieres que jadis en avoient les dis rommains souverainement politiques en toutes choses, c'est assavoir que les dis offices fussent donnéz et distribuéz, non mie par telz simonies ou faveurs, mais par pure, loyalle et vraie eleccion par preudommes bien congnoissans et par bonne enqueste donnez aux plus vallables et mieulx dignes [...]. (Paix, p. 82).

Ici, le terme d'office est opposé implicitement aux principes démocratiques mêmes, puisque Christine place en face des pratiques des Romains celles qu'elle juge déplorables, à savoir simonies et faveurs. Mais ce qui est remarquable, c'est l'habileté avec laquelle Christine insère les notions démocratiques telles que celle de citoyen, bien représentées pour politiques ${ }^{22}$.

Il arrive que office soit déterminé par un complément qui spécifie le type de "charge » ou de "fonction». Ainsi, office finit, chez Christine, par entrer dans le champ sémantique du doublet rhétorique sapientia et fortitudo, qui a traversé toute la littérature épique et courtoise du XII ${ }^{e}$ siècle; mais ce qui semble plus caractéristique encore de la pensée de Christine est la prévalence qui est accordée au sens (« intelligence ») sur l'éventuel prestige de telle ou telle fonction :

Et contre ceulx qui dient les honneurs changent les meurs, ilz ne donnoient mie les offices ains que le sens fust venus ains actendoient le sens estre venus devant, et mesmement es offices d'armes les plus sages et les plus excitéz les avoient, car ne leur souffisoit mie que homme fust seullement bon de la main se sens d'armes n'estoit avec, comme ilz tenissent que plus prouffitoit mesmes es fais de chevalerie sens que force, et se aucun eust fait quelque entreprise follement et hors ordre de droit d'armes et de raison et toutesvoies bien en fusr ensuivy au chevetain pourtant 
en a cellui qui l'avoit faicte ne lui eust ja estre atribuée à honneur, mais à aventure.

(Paix, p. 82).

Un nouveau concept prend forme à travers la définition de l'office idéal, à savoir celui de sens d'armes, une intelligence stratégique ou une intelligence de stratège. Christine avait déjà, mais plus discrètement, abordé la question dans Charles $V^{23}$. Désormais, même si elle n'est pas vraiment la première à évoquer la question, la prouesse ${ }^{24}$ et les fais de chevalerie entrent dans une sorte de phase rationalisation et seront soumis à un nouvel ordre, l'ordre de droit d'armes ${ }^{25}$. Une réglementation juridique présidera - du moins idéalement - aux faits d'armes. L'adverbe mesmement montre bien que c'est le cas extrême et que, à plus forte raison, dans les autres domaines de la vie politique, c'est le sens qui devra être un des critères dans le choix des hommes à telle ou telle fonction. L'office, la façon de s'en acquitter, se situe à présent à l'opposé de l'aventure, de la folle aventure (eust fait quelque entreprise follement).

Aussi, dès la Mutacion de fortune, constate-t-on chez Christine que l'office est loin d'être attribué à tort et à travers, sans discernement :

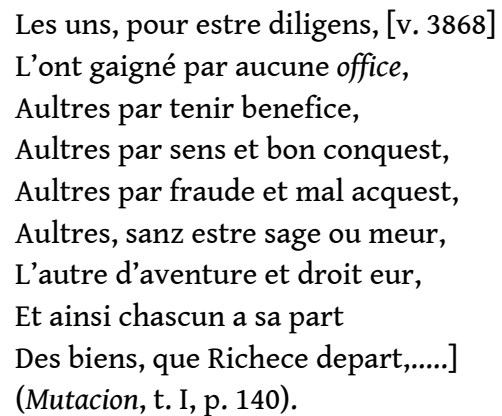

Il en est de l'office comme de toute chose: ce qui est louable côtoie ce qui est méprisable, mais le premier cas existe bel et bien. Aussi benefice n'a-t-il pas forcément une connotation péjorative.

31 Toujours dans la Mutacion - et on comprend que ce soit ici plutôt que dans une autre œuvre - office, pouvant osciller soit du côté positif, soit du côté négatif, peut glisser vers le champ sémantique du devoir, devoir bien fait, devoir mal fait, bien que la notion même de « devoir » ne soit pas un sémème caractéristique de l'aire sémantique d'office:

Se leur office loyaument [v. 3933]

Font, sanz mener trop rudement

Le menu peuple besongneux,

Et sont diligens et songneux

De faire en tous cas leur devoir,

Preu et loz en doivent avoir,

Mais, s'aultrement font, ilz se perdent

Et les dampnent les biens qu'ilz gardent.

(Mutacion, t. I, p. 142).

Le contexte est particulièrement éclairant ici, puisque font leur office semble être concurrencé par faire leur devoir ${ }^{26}$. D'ailleurs, on constate ici et ailleurs que office a tendance à entrer dans des réseaux contextuels dominés explicitement par des termes appartenant au vocabulaire moral; il en est ainsi dans le passage cité où office et loyalment ${ }^{27}$ font l'objet d'une collocation, ce qui montre bien que le sémantisme d'office, fait l'objet d'une intériorisation et d'une moralisation. 


\section{II.- Relations et société}

33 Tout le réseau lexical qui fait l'objet de cet examen repose sur la notion essentielle de relations humaines, sans lesquelles la société et le tissu social n'existeraient pas.

\section{Communicacion et communité}

Dans le vocabulaire politique et éthique, deux termes, inexistants dans la langue de l'ancien français, communicacion et communité, jouent, dans l'organisation des textes du $\mathrm{XIV}^{\mathrm{e}}$ siècle, un rôle de première place. Ce sont deux termes dont le noyau sémantique repose sur le sème "collectivité » ou " hommes vivant ensemble », mais il s'y ajoute incontestablement le sème virtuel relevant de la connotation politique au sens étymologique de ce terme, à savoir " ayant trait à la vie de la cité », notion elle-même située dans le cadre de la doctrine sociale et politique d'Aristote.

\section{Communicacion}

Le terme prend une ampleur toute particulière dans l'œuvre d'Aristote traduite par Oresme. Le vocable ${ }^{28}$ recouvre chez Oresme habituellement les valeurs suivantes, dont l'archisème est incontestablement « relation »:

\section{$1^{\circ}$ « Relations (humaines) »}

Et pour ce selon une autre maniere, choses justes legals proprement sont celles par quoy felicité est faite ou gardee et chascune de ses parties en communicacion civile. (Éthiques. Texte, t. II, p. 278).

Définition qui englobe des aspects qui relèvent à la fois de la morale et de la politique. En effet, c'est d'abord la notion de justice morale qui est liée à celle de légalité politique et sociale ; pour Aristote, l'un ne va pas sans l'autre ; le bonheur lui-même (felicité) est conditionné par cette harmonie, qui à son tour, relève forcément des relations (communicacion) entre citoyens (civile), avec, à l'arrière-plan, la cité elle-même, cité ou policie?

Quelquefois, le domaine dans lequel se placent ces relations est précisé par un complément déterminatif :

Car toutes trois [vertus] $]^{29}$ sont en communicacion de paroles et de operacions.

(Éthiques. Texte, t. I, p. 167).

Aristote met l'accent sur les moyens mis en œuvre dans les relations humaines, qui sont le discours (paroles) et l'acte, voire l'activité (operacions). De façon concrète, le discours et l'acte, surtout l'acte politique, sont étroitement liés l'un à l'autre. C'est un des principes même de la démocratie athénienne, et il est intéressant de constater la manière dont, ici, Oresme, manifestement à l'intention de Charles $\mathrm{V}$, insiste sur la primauté de la parole, du discours, entendu comme le discours entre citoyens et, donc, aussi entre le roi et ses sujets. En somme, la morale de l'individu et celle de la société ne sauraient avoir d'effet en dehors de la parole.

Il arrive que le complément prépositionnel porte sur des personnes :

Et pour ce convient il toutes choses aprisier a monnoie et en ceste maniere se fera tousjours commutacions, et par consequent sera communicacion entre les gens.

(Éthiques. Texte, t. II, p. 297). 
Dans ce même ordre d'idées, on relève les syntagmes communication humaine ${ }^{30}$ et communication politique. C'est certainement cette dernière expression qui est la plus éloquente quant au renouveau qu'Oresme apporte au discours politique en français du XIVe siècle :

Aprés il entent a monstrer une autre conclusion, c'est a savoir, que toute communicacion est reduit [sic] ou ramenee a communicacion politique. (Éthiques. Commentaire, t. II, p. 432).

41 Ce syntagme comporte une valeur légèrement différente de celle de communication civil, en tout cas une portée bien plus large que cette dernière expression, puisque politique fait référence, incontestablement, à l'organisation de la cité et, dans une moindre mesure, à la cohésion des citoyens. Il est vrai que, dans les deux cas, il est question, explicitement ou implicitement, d'un corps social. D'ailleurs, un peu plus loin dans le même texte, il est dit très clairement que les relations entre hommes ne sauraient se situer en dehors de la Cité et de ses exigences :

Et toutes communicacions ont similitude a aucunes parties de communicacions. Car toutes toutes conviennent en aucune chose conferente et utile pour acquerir aucune chose neccessaire ou convenable a vie humainne. Et communicacion politique est ordenee au commencement, et persevere pour grace de conferent et de utilité publique. [...] Et avec ce tous dient que querir le commun proffit est juste chose. (Éthiques. Texte, p. 432).

\section{$2^{\circ}$ "Communication d'un lieu avec un autre"}

Apparemment cette seconde valeur semble fort éloignée de la première. En fait, l'aspect relationnel est toujours explicitement présent :

Aucuns funt doubte de la communication que cité peut avoir a la mer, assavoir mon se elle est profitable ou se de ce viennent choses nuisibles. (Politiques. Texte, p. 295b).

La question de l'utilité et du profit publics est d'emblée posée par Oresme, et c'est la cité qui est le centre d'intérêt. D'ailleurs, le contexte large ${ }^{31}$ dans lequel s'insère ce passage montre clairement que ce qui préoccupe Aristote - et Oresme dans son commentaire ${ }^{32}$ abonde dans ce sens - c'est bien l'ouverture ou la fermeture de la cité sur le monde extérieur et que c'est, en particulier, le problème éminemment relationnel de l'immigration qui est au premier plan du débat engagé.

Que ce soit dans le prétendu texte d'Aristote ou dans le commentaire d'Oresme, partout, c'est la notion de communauté ou de collectivité, soit l'intérêt commun, qui est présenté comme le fondement même de toute vie organisée à l'intérieur de la Cité.

Alors que le terme est bien représenté chez Oresme, il est rare chez Christine. Dans le corpus des œuvres saisies intégralement par l'INaLF ${ }^{33}$, on peut relever que deux occurrences appartenant toutes deux au Livre de la Paix, et chaque fois le sème " homme » est présent d'une manière ou d'une autre. Dans l'un des deux exemples, c'est le syntagme communicacion humaine qui entre dans une définition :

Et lui meismes dit, si que allegué est cy dessus suivant ou latin: Communicacion humaine est de la lanture de tout bien. (Paix, p. 178).

Dans l'autre, on trouve la variante communicacion avec les hommes:

Mais quoy que communicacion avec les hommes soit bonne, n'est mie a entendre de ceulx qui sont mauvais, car de tel gent n'est compaignie bonne ne belle, si que assez est dit devant. (Paix, p. 178). 


\section{Communité} entre vraiment, grâce à lui, dans le domaine politique. En effet, avant Oresme, quelques emplois timides de ce terme se manifestent dans le dernier tiers du XIII ${ }^{e}$ siècle, notamment dans des œuvres du début du XIII ${ }^{e}$ siècle $^{35}$.

\section{a) Chez Oresme}

Dès que le terme apparait dans l'œuvre d'Oresme, il prend une connotation politique dans le cadre des valeurs suivantes :

\section{$1^{\circ}$ "Société »}

Le sens général de "société » que peut comporter le terme est en fait orienté vers la notion de vie commune entre citoyens dans le cadre d'une organisation de la cité. Pour preuve, Oresme commente dans le social et politique même un comportement qui, à première vue, relève simplement du caractère ou du tempérament individuel :

Et celui qui fuit tous tels deliz de corps, il est agreste et insensible. (Éthiques. Texte, t. I, p. 150)

Ce passage est glosé de la manière suivante :

Il est a entendre de celui qui vit en communité et de vie politique ou civile ; car, se il ne prent nullement aucune delectacion corporel, c'est par deffaute de bonne nature ou par mauvaise et fole acoustumance (Éthiques. Commentaire, t. I, p. 150).

L'enchaînement logique entre les deux passages cités, le texte et la glose, mérite une explication en ce sens que le rapport entre agreste/insensible et celui qui vit en communité n'est pas évident. Oresme, dans sa glose, tient précisément à indiquer que la notion contenue dans les deux adjectifs est à prendre en compte dans le cas particulier où les citoyens auraient le comportement désigné par ces adjectifs, et ce dernier représente, aux yeux d'Oresme, une situation d'exception. Oresme montre clairement ce qu'il en pense en donnant la cause de cette déviation: deffaute de bonne nature et mauvaise et fole acoustumance. C'est donc une mauvaise éducation et un mauvais tempérament ou caractère qui sont à l'origine de cette attitude asociale; car, en fin de compte, agreste signifie "qui a un comportement de rustre ", donc qui se montre asocial, et insensible ${ }^{36}$ qualifie l'homme qui se montre stupide, voire inadapté, dans le milieu social dans lequel il vit.

\section{$2^{\circ}$ "Relations "}

Oresme, lui-même, dans une glose, précise le rapport qui existe entre les citoyens (communité civile) et correspond à un type de relations humaines (communicacion) :

Tele communicacion est communité civile ou l'en doit trouver tout ce que est neccessaire et convenable a vie humaine, selon commune posibilité. (Éthiques. Commentaire, t. II, p. 300).

51 Ce sont les relations entre citoyens qui permettent l'existence même de la vie humaine, qui repose sur une entr'aide mutuelle (selon commune posibilité). La survie de l'homme se situe nécessairement dans la collectivité. 


\section{$3^{\circ}$ " Collectivité "}

52 Aussi n'est-on pas surpris de voir le terme affecté du sémème " collectivité ", notion générale, sans qu'une caractérisation ou une complémentation quelconque spécifient le type de collectivité :

Et monarchie, c'est la ou .I. tout seul a souveraine seigneurie sur une communité.

(Éthiques. Commentaire, t. II, p. 434)

La locution en communité (« collectivement »), dans ces conditions, se trouve associée à civilement :

[...] car homme est par nature ordené a vivre civilement et en communité. (Éthiques.

Commentaire, t. I, p. 118).

\section{$4^{\circ}$ « Communauté (en tant qu'unité politique) »}

54 L'aspect proprement politique du regroupement des hommes peut être exprimé de façon plus ou moins explicite. Lorsque l'explicitation contextuelle atteint son plus haut degré, le terme désigne l'organisme politique lui-même :

Pour ce pent l'en les larrons afin que la policie ou la communité en soit purgiee, et afin que les autres se corrigent par tel example, et que la painne de un face paour a pluseurs. (Éthiques. Commentaire, t. II, p. 533).

Dans ce texte, qui est une glose d'Oresme, une adéquation est établie entre communité et policie dans le sens de "organisation de la cité » ou «organisme politique ». Il est évident que l'arrière-plan du passage cité est dominé par une préoccupation morale (purgiee, corrigent), qui repose sur des contraintes ou des règles.

\section{b) Chez Christine}

L'emploi du terme est relativement rare chez Christine, et presque chaque fois, il entre dans un réseau contextuel tel qu'il est soutenu par des vocables à connotation collective, sociale ou politique. Ainsi, dans le Livre des fais et bonnes meurs du sage roy Charles $V$, il s'intègre dans la définition des vocables ville et cité $e^{37}$ :

[...] dont, se l'en demande quel chose est ville, on doit dire que villes et citez ne est fors que une assemblée assemblée et une communité de gens establie pour bien et vertueusement vivre et selon raison [...]. (Charles V, t. II, p. 27).

57 L'organisation rationnelle (selon raison) et morale (vertueusement) vise un état sinon définitif du moins destiné à durer (establie), de cette « collectivité » qu'est la communité. D'ailleurs, il arrive que l'idée même de l'action de gouverner soit directement attachée au terme qui devient alors synonyme de policie. Dans le Livre de la paix, c'est une bonne partie de l'arrière-plan de la vie politique et sociale qui sous-tend l'emploi du terme :

En autres estas sont compris clers et laiz, c'est assavoir clers legistes et autres sages, soient prelaz ou autres, ausquelz les lois aient apris droit de gouverner policie et communité de toutes manieres d'estas de gens. (Paix, p. 76).

En effet, Christine insiste bien sur l'idée de non-exclusivité, d'universalité humaine qui caractérise la communité, puisque tous les milieux professionnels et sociaux sont concernés (toutes manieres d'estas de gens) et en constituent le tissu politique et social. Bien plus, ce passage montre combien, à l'époque de Christine, la réglementation devient l'affaire de la communauté ${ }^{38}$, de l'organisme politique qui régit les hommes soumis à un même régime. 

l'organisation politique longuement mûrie s'effondrent par la faute de quelques-uns :

Riens n'est plus espouventable en cité ne plus perilleux en communité que homme sedicieux et raporteur, et qui de cellui tient parolle fait ainsi que s'il gectoit huille en un feu tres perilleux pour plus l'atiser. (Paix, p. 171).

La force de l'image à laquelle recourt Christine laisse entrevoir la nature de la nouvelle vision de l'État qui se fait jour à la fin du XIV et au début du XV ${ }^{e}$ siècle. La conception de l'État souverain est néanmoins tempérée par la lucidité de Christine, qui, ailleurs dans le Livre de la Paix, apporte une réserve à sa vision du monde :

[...] comme chose soit impossible tenir et garder une grant communité que pluseurs d'eulx par simplece, mauvais conseil, ou autrement, n'en courrussent maint deffaulx veu nature humaine estre de soy encliné à tous vices [...]. (Paix, p. 117).

\section{Multitude et ses coordonnées}

61 Un terme qui prend une coloration nouvelle dans la seconde moitié du XIV siècle est bien celui de multitude, tout particulièrement dans l'optique dans laquelle se placent des auteurs tels qu'Oresme et Christine de Pisan.

\section{a) Chez Oresme}

62 Le terme peut prendre, dans les Politiques, un sens contextuel bien particulier dans l'optique de la pensée aristotélicienne ou, en général, athénienne.

\section{$1^{\circ}$ «Peuple »}

63 Cette notion intervient à propos de la question que se pose Aristote : le peuple doit-il élire ou, éventuellement, mettre dans le droit chemin le prince? En raison de l'ignorance et de l'incompétence du peuple, Aristote émet une réponse négative :

Et donques selon ceste raison l'en ne doit pas faire que la multitude ait domination ne qu'elle ait le election des princes ne les corrections. (Politiques. Texte, p. 136b).

Et Oresme, dans la glose, d'ajouter :

Ceste raison suppose que la multitude ne ait pas science ne art de gouverner ou de emender la policie. (Ibid.).

Dans la notion de "peuple" s'insère, qu'on le veuille ou non, une connotation péjorative ${ }^{40}$, qui, d'une certaine manière, laisse le champ libre à la suprématie modérée et raisonnée du prince.

\section{$2^{\circ}$ «Assemblée du peuple »}

Par métonymie, le terme glisse vers la notion de " peuple assemblé » et " assemblée du peuple » avec, cette fois, une valeur méliorative. Le contexte est tel que multitude est une sorte d'anaphore de tous assemblés. Cet emploi est d'autant plus intéressant que le second syntagme figure dans le texte proprement dit, et le premier, dans la glose correspondante :

Car se la multitude ne est trop ville, combien que chescun singulier soit moins bon juge que ne sunt les sachans, nientmoins tous assemblés sunt melleurs juges que un petit nombre de gens sachans ou au moinsn ne sunt il pas pires juges. (PolitiquesTexte, p. 136b)...] Et donques l'intention de Aristote est, ce semble, que tele multitude doit avoir l'election des princes et la correction de leur fais. (Ibid.- Glose). 
Il semble bien clair qu'il est question du peuple qui siège pour juger et pour élire éventuellement le prince, et que, par conséquent, multitude se trouve affecté du sémème « assemblée du peuple ».

\section{b) Chez Christine}

67 Cette connotation politique et sociale de multitude a pratiquement - semble-t-il ${ }^{41}$ disparu de l'œuvre de Christine, où le terme n'est affecté que de la valeur "grand nombre de » avec un complément déterminatif animé ou inanimé. Souvent, multitude est caractérisé par l'adjectif grant :

[...] et dit le Philosophe que reaume n'est que une grant multitude de gens, qui vivent selon loy et vertu, et sont ordennez soubz un chief, qui vertueux doit estre, et à lui apertient obeissance de tous, et doit ce roy diligement pener que selon raison et loy ses subgiez se gouvernent et soient gouvernez. (Charles $V$, t. II, p. 28).

Il est vrai qu'ici, le terme figure dans un contexte qui se réfère à quelques notions politiques et morales. Néanmoins, il est confiné, quant à sa valeur proprement sémantique, dans la notion de « nombre » pure et simple.

\section{III.- La cité}

Mais, après tout, cette dernière notion n'est pas très éloignée de la valeur d'un terme qui, lui, aussi bien chez Oresme que chez Christine, comporte un dénotation politique et sociale, à savoir cité.

\section{a) Chez Oresme}

Bien que ville et cité aient tendance en moyen français de devenir équivalents, il arrive que cité ait gardé la valeur initiale de « ville fortifiée ». De fait, dans la Table des notables (p. 359, éd. A.D. Menut) :

Que l'en doit garder les murs et les portes des cités en temps de paes et en temps de guerre. (Politiques- Glose, p. 359b) ${ }^{42}$.

71 Un emploi plus fréquent est celui où, entre ce terme et celui de citoien, se tisse un rapport de contenant à contenu, dans la mesure où cité signifie l'ensemble des citoyens :

Item, se une chose est bonne a un citoien laquelle est bonne a toute la cité et aussi se ce qui est bon a toute la cité est bon a un citoien. (Éthiques. Texte, t. I, p. 105).

Quand il s'agit des idées d'Aristote transposées au XIV siècle, le terme de cité prend nécessairement une connotation politique bien particulière, celle de la cité athénienne $\mathrm{du} \mathrm{V}^{\mathrm{e}}$ ou du IV $\mathrm{IV}^{\mathrm{e}}$ siècle avant J.-C. Cité devient alors une entité politique. D'ailleurs, sans le Livre des Éthiques, les termes de païs et de cité se trouvent mis sur le même plan, ce qui montre - si besoin était d'y insister - qu'Oresme connait parfaitement l'acception du terme de civitas, qui peut dépasser la sphère de "ville » et approcher de la notion d'« État ", au moins au sens territorial :

Ne le gouverneur de la policie du païs ou de la cité ne conseille pas se il fera ou querra bonne paix en son peuple. (Éthiques. Texte, t. I, p. 191). 
Ici, la cité est impliquée dans une situation de paix ou de guerre, ce qui éloigne le terme de la sphère de la simple relation entre citoyens, puisque c'est ce qui est extérieur à la cité qui est confronté à ce qui est intérieur à cette même cité.

Cette opposition est bien mise en évidence dans le passage suivant :

Que usage d'armes est necessaire en cité pour .ii. causes : pour faire obeïr les siens a

justice, et pour les garder et deffendre des estranges [...]. (Politiques. Glose, p. 359b).

Les divers aspects de la cité sont représentés conjointement et replacés dans le cadre de la politique intérieure et extérieure.

\section{b) Chez Christine}

En ce qui concerne l'œuvre de Christine de Pizan, il convient de distinguer soigneusement le genre littéraire où figure le terme. Cette précaution s'impose à qui connaît la variété de son œuvre.

\section{$1^{\circ}$ Le Livre de la mutacion de fortune}

76 Cette œuvre présente des emplois de cité relativement stéréotypés. Toutefois, plusieurs tendances se font jour.

- Un environnement contextuel valorisant

Nombreux sont les cas où le terme de cité est valorisé par la présence d'un nom de cité antique prestigieuse :

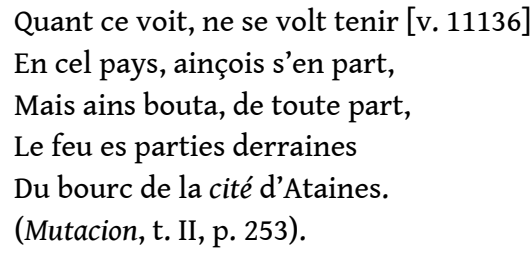

78 C'est le nom d'Athènes qui, à lui seul, rehausse en quelque sorte la valeur connotative du terme.

- Mais le contexte peut être valorisé par des noms communs qui contribuent à faire de la cité un point de mire pour les hommes qui la trouvent sur leur chemin :

Moult fu roy Assurus poissant [v. 11281]

Et tous les jours aloit croiscent

Sa force, ce nous dit l'istoire.

Si aloit, demenant sa gloire,

En sa maistre cité nommée

Sussis, qui tant fu renommee.

(Mutacion, t. II, p. 258).

Gloire et renommee ${ }^{43}$ sont des coordonnées particulièrement éloquentes; mais c'est encore plus l'adjectif maistre qui confère une importance à la cité évoquée. Cité est précédé de termes laudatifs (poissant, croiscent, force).

- Le contexte peut être encore chargé d'éléments emphatiques

[Le roi Assuaire lance un appel à tous ses barons]

Et tuit venissent sanz sejour [v. 11338]

En la grant cité de Sussis,

Car la tendroit, a jour pressis,

Court banie et feste planiere.

(Mutacion, t. II, p. 260). 
- Extension de la valeur spatiale de cité, « capitale » d'un royaume :

Cette valeur de "capitale" (chief $)^{44}$ peut elle-même être justifiée par toute une description laudative de la cité, et dans le passage présenté ci-dessous, on croit constater une gradation d'importance croissante de ville à cité :

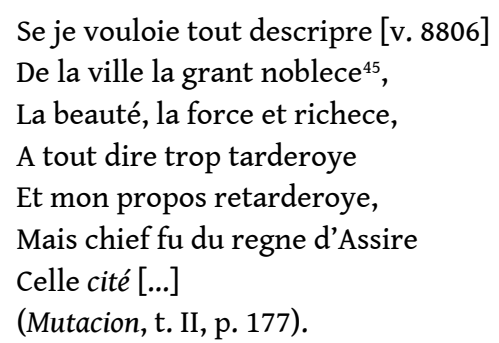

81 C'est tout particulièrement le syntagme La beauté, la force et richece qui rehausse le tableau qui nous est donnée par Christine. Quant à la formule de prétérition, elle est fort habile ici, puisqu'elle laisse le lecteur en haleine : il souhaiterait en savoir encore davantage.

\section{$2^{\circ}$ Dans le Livre du corps de policie}

Dans ce texte, où les considérations politiques sont nécessairement nombreuses, le terme de cité, dans son emploi, se rapproche singulièrement de cité employé dans les Politiques d'Oresme.

\section{Cité et pays}

Cité comporte ici une valeur quelque peu ambiguë, celle de "ville fortifiée, capitale d'un pays en tant que territoire politiquement délimitée » :

Car a deffendre la cité et le pays vint si grande quantité des femmes qu'il couvient a Pirrus s'en partir honteusement [...]. (Policie, p. 55).

Il est rare qu'on soit ainsi en présence, avec ce terme, d'une conception relativement moderne du rapport de la ville par excellence d'un État à l'ensemble de l'État; le patriotisme ici évoqué ne fait que renforcer la cohésion de cet unité politique ${ }^{46}$.

\section{"Ville fortifiée" "}

Il arrive que l'acception première ${ }^{47}$ du terme de cité « partie la plus ancienne d'une ville et, donc, en tant que noyau historique, la plus fortifiée $»^{48}$, qui avait pratiquement disparu de l'aire sémantique du même terme, resurgisse ; dans l'exemple présenté cidessous, c'est précisément cette partie ancienne de la ville qui est spécifiquement désignée par le nom propre adéquat, à savoir le Capitole, quand il s'agit de Rome. Ce glissement est d'autant plus significatif qu'il intervient dans le récit des anecdotes que rapporte Christine à travers, il est vrai, la plupart du temps, des historiens ou chroniqueurs latins, notamment Valère-Maxime :

Et par ce il desprisa les paroles d'ung saige chevalier qu'on nommoit Maharbal, qui ses oreilles oans disoit et affermoit qu'il avoit avisé par quele maniere en pou des jours Hanibal prendroit Romme et souperoit ou capitole qui estoit la maistresse forteresse de la cité, si n'y daigna entendre et n'en tint compte. (Policie, p. 59). 


\section{"État démocratique»}

86

Un pas de plus dans le sens de l'abstraction politique se trouve dans l'emploi de cité aux côtés de majestés royaulx (« souveraineté royale » au sens de «souveraineté territoriale d'un royaume »); cette opposition conduit au sens antique de civitas "État », et s'ajoute alors, par contraste avec royaulx, la connotation « démocratique » :

Car par euvre de prudence es consaulx des vieillars sont soustenus et gouvernés les majestés royaulx et les cités, policies et choses publiques qui souvent sont mises au bas par les jeunes, si comme dit Tulles [...] (Policie, p. 65)

L'apposition policies et choses publiques indique bien qu'il est question, avec les deux dénominations, de types de gouvernement et de types d'État. Ces gouvernements requièrent pondération et équilibre, qui ne sont pas le propre de la jeunesse ${ }^{49}$.

\section{« État » au sens territorial pur et simple}

Un autre cas se présente où, toujours dans Policie, cité est affecté de la valeur d'« État » au sens territorial. Le terme se trouve appuyé par une coordonnée contextuelle de première importance dans ce cas, à savoir peuple :

Car selon ce que dit Archica de Tarente qui fut tres grant philosophe, nulle plus capitale pestilence n'est donnee a l'omme par nature que volupté corporele, de la quele naisent traisons, submersions des cités et des peuples, violences et tous maulx. (Policie, p. 66).

\section{«Peuple»}

Dans un dernier élargissement, cité, par un processus métonymique, recouvre les hommes vivant dans la cité au sens d'«État ", et ainsi c'est la valeur connotative de "peuple $»^{50}$ qui semble se dégager de l'emploi contextguel de cités1.

[A propos d'un vieillard qui, en tant que tel, a été honoré par les notables de Lacédémone, après avoir été méprisé par les gens du peuple d'Athènes]

[...] si ala tant qu'il vint d'aventure ou lieu ou les legas, comme nous dirions ambassadeurs, de Lacedemone seoient, qui estoient venus en message a Athenes, lesquelz estoient jeunes d'aage, et tantost selonc leur coustumes honnourerent le vieillart, et tous se leverent et lui baillerent honnourable lieu entre eulx. Et quant le peuple vit ce il approuva moult la noble coustume de l'extrange cité, et dirent entre euls nous sçavons bien qu'est droit, mais nous nous en attandons aux estranges. (Policie, p. 69).

Le contexte est tel que l'opposition n'est pas tant entre les deux cités d'Athènes et de Lacédémone qu'entre les deux peuples respectivement concernés. L'adjectif extrange ne fait que renchérir sur cette opposition.

\section{$3^{\circ}$ Le Livre de la paix}

91 De plus en plus souvent, le terme glisse du côté de la notion d' «État ", qui comporte d'un côté le peuple (populaires), de l'autre côté les notables, ceux qui, précisément, sont dignes d'occuper des charges officielles (offices):

Doncques, pour les raisons susdictes, si que ou chapitre precedent est touchié, office de cité n'appartient aux populaires [...]. (Paix, p. 132).

C'est encore dans ce sens qu'il convient de comprendre l'emploi du même terme, lorsque estat (au sens de « situation ») vient s'adjoindre à cité : 
Et comme jamais ne leur souffise, quelz que bons gouverneurs que ilz aient, vouldroient tousjours que estat de cité se rechangiast. (Paix, p. 133).

$1^{\circ}$ L'importance accordée à la notion de gouvernement entraîne la création de policie dans l'œuvre d'Oresme et Christine en fait le titre d'un de ses livres.

$2^{\circ}$ Avec les termes de communité et communicacion, la réflexion d'Oresme accorde de plus en plus d'importance à la démocratie ou l'État démocratique; pour preuve, on peut avancer le fait que multitude prend une connotation nouvelle dans la mesure où il désigne la totalité des habitants de la cité en tant que citoyens. Oresme semble transposer le terme dans la civilisation médiévale ; en effet, il n'évoque pratiquement pas les catégories sociales qui, dans l'Antiquité, ne pouvaient être citoyens. En somme, dans l'esprit d'Oresme se forme un amalgame entre la notion de peuple dans un sens démocratique et celle de sujets dans un État monarchique.

$3^{\circ}$ Dans ces conditions, le terme de cité connaît un singulier glissement, puisqu'il finit par désigner l'État.

Quant à la différence pouvant exister entre l'usage d'Oresme et celui de Christine, elle est de l'ordre de la capacité d'intégration de ces termes dans les structures mentales de la société de la fin du XIV siècle. Dans cette optique, il faut reconnaître que l'emploi qui est fait de ces termes ${ }^{56}$ par Christine a perdu en technicité ${ }^{57}$ par rapport à ce qu'ils sont chez Oresme, ils ont gagné en modernité et en efficacité dans la représentation de la société. Mais un écart bien plus significatif semble se dessiner entre le discours d'Oresme et de Christine, notamment au plan proprement idéologique : Oresme, sans 
doute de façon discrète, mais non moins réelle, met l'accent sur l'harmonie qui doit exister entre celui qui gouverne et les sujets, voire le peuple entendu dans le sens politique d'Aristote; qu'on le veuille on non, certains aspects de la démocratie athénienne envahissent des concepts proprement médiévaux ou, du moins, qui l'étaient jusque-làj ${ }^{58}$, plus dans l'œuvre d'Oresme que dans celle de Christine. Toutes proportions gardées, la notion de bien commun sous-tend, de façon identique, la vision $\mathrm{du}$ monde des deux auteurs ${ }^{59}$. Si cette vision semble plus proche des réalités contemporaines chez Christine ${ }^{60}$, l'œuvre d'Oresme, et tout particulièrement les Politiques, sont loin d'ignorer la situation politique et sociale contemporaine ${ }^{61}$; ainsi, il n'hésite pas à prendre parti dans la question du favoritisme ${ }^{62}$ et dans celle du gallicanisme ${ }^{63}$, ce qui confère à son vocabulaire politique et social une tonalité différente de celui de Christine de Pizan.

\section{NOTES}

1. Cf. sur ce point l'article de L. Dulac, Unité et variations de la sagesse dans le « Livre des fais et bonnes meurs du sage roy Charles V", Revue des langues romanes, t. 92 (1988), pp. 296-307.

2. Le terme peut prendre une acception générale se situant en dehors du champ politique et social. Cf. J. Blanchard et M. Quereuil, Lexique de Christine de Pizan, Paris, Klincksieck, 1999 (Matériaux pour le Dictionnaire de Moyen Français, 5), p. 302.

3. Il s'agit d'un mot-clé de haute fréquence. Cf. S. Lusignan, Indexer et gloser: Nicole Oresme et la «Politique » d'Aristote, L'Écrit dans la société médiévale. Divers aspects de sa pratique du XIe au XVe siècle, Textes réunis par C. Bourlet et A. Dufour, Paris, CNRS Éditions, 1993, p. 172-73.

4. On peut constater la souplesse des équivalences proposées. Oresme veut-il dire que polis et civitas peuvent dépasser le cadre proprement topographique de la « ville (fortifiée) »? En effet, la cité était la plus petite circonscription romaine, subdivision de la province; voir Vocabulaire historique du Moyen Âge (Occident, Byzance, Islam) sous la dir. de Fr.-O. Touati, Paris, La Boutique de l'Histoire, 1997, sous cité, p. 67-68.

5. Maistre Nicole Oresme. Le Livre de Politiques d'Aristote, éd. A. D. Menut, Philadelphia, The American Philosophical Society, 1970 (Transactions of the American Philosophical Society, vol. 60).

6. Ce terme indique qu'il s'agit d'un discours propre à Oresme; le terme de texte signifiera qu'Oresme entend traduire le texte d'Aristote. Ce terme de glose peut englober, comme c'est le cas ici, la Table des expositions, qui ne figure évidemment pas dans l'ouvrage d'Aristote.

7. Le terme de cité n'est pas mentionné ici en relation avec policie; en revanche, dans le réseau contextuel de cité figure communité, qui sera pris en compte plus bas.

8. Gouverneur a encore le sens général de «celui qui préside à qqc. » Cf. FEW IV 301a gouverneur « administrateur ecclésiastique, directeur d'un établissement public ». Le sens premier, il est bon de le rappeler, est « celui qui est chargé de la conduite d'un bateau » (op. cit., p. 299b).

9. Maistre Nicole Oresme. Le Livre de Éthiques d'Aristote, éd. A. D. Menut, New York, Stechert, 1940, 2 vol. On distinguera entre Texte et Commentaire.

10. The Livre de la Paix of Christine de Pisan, éd. Ch. C. Willard, Gravenhage, Mouton, 1958.

11. Christine de Pizan. Le Livre des Trois Vertus, éd. Ch. C. Willard et E. Hicks, Paris, Champion, 1989.

12. Au sens de « ordonnance, agencement». 
13. Le Livre des fais et bonnes meurs du sage roy Charles V, éd. S. Solente, Paris, Champion, 2 vol., 1936-1940.

14. C'est le cas dans l'administration politique de l'Europe occidentale du XIV e siècle. CF. B. Guenée, L'Occident aux XIV et XV siècles, Paris, P.U.F., 1971, p. 277.

15. Au sens de "procédure, réglementation ». On peut signaler que c'est en moyen français surtout qu'apparaît ce genre d'emploi.

16. Il suffit d'évoquer les Politiques d'Oresme ou le Songe du vergier.

17. Le Livre du corps de policie, éd. R.H. Lucas, Genève, Droz, 1967 (T.L.F., 145).

18. Il s'agit d'une glose. Donc, c'est Oresme qui émet son avis personnel sur la question au delà du texte même d'Aristote.

19. Ce terme a aussi, dans les Éthiques et dans les Politiques, deux sens, qui sont étroitement liés à celui de « charge », « domination » et « principauté (en tant que territoire) ».

$1^{\circ}$ «Domination » dans la locution avoir princé : Et pour ce, en gubernacion ou policie juste et droiturière, nous ne laisson ou souffron quelconquez personne ait princei, doinacion ou seigneurie selon homme, mais elon raison et selon la loy mise. (Éthiques. Texte, t. II, p. 301). La collocation gubernacion/policie mérite d'être remarquée.

$2^{\circ}$ «Principauté (en tant que territoire) »: Le tiers [point est], car ilz distribuent les princés et dominacions a uns meïsmes tousjours. (Politiques, p. 138b).

De la première valeur semble dériver le sens de « charge ».

20. Dans la Base des Textes de Moyen Français (INaLF), princey/princei/princé n'est pas signalé pour les œuvres de Christine retenues.

21. Livre de la mutacion de Fortune, éd. S. Solente, Paris, Picard, 4 vol., 1959-1966 (SATF).

22. Le terme, de par son étymologie (en grec, politikos est un dérivé de polis « la cité »), concerne avant tout les citoyens, leur place dans le déroulement des affaires publiques. L'adjectif est affecté du sens de "utile au gouvernement de la cité », " habile dans le gouvernement de la cité ".

23. Cf. article de L. Dulac, voir note 1 .

24. Les expressions bon de la main et force sont significatives dans ce réseau d'oppositions.

25. Il faut comprendre : « les règles de la juridiction chevaleresque ».

26. Dans la langue des auteurs d'écrits à orientation politique, il arrive que office dans ce sens soit déterminé par un complément déterminatif, qui précise alors la teneur du devoir. Ainsi, chez Denis Foulechat, Policratique, Livre IV, chap. 6, p. 66 (éd. Ch. Brucker, CERES) : «Je me recorde avoir leu ou livre Noctium acticarum que en lisant les noblesces des bonnes meurs de Phelippe le roy de Macedoine, il est escript que entre touz ses fais il laboura moult et s'estudia de embelir et colorer l'estude des clers et personnes lettrees aussi comme les fais de ses batailles et les honneurs de ses victoires et liberalité de sa table et office de humanité et toutes choses dittes ou faittes bel et plaisaument. »

27. La collocation en question prouve - si besoin était - que la connotation morale d'office n'est jamais complètement absente.

28. L'analyse qui suit est empruntée à notre lexique des œuvres d'Oresme (à paraître).

29. Le contexte indique qu'il s'agit de magnanimité, phylotimie, manuetude (ou debonnaireté).

30. «Et sont toutes [les trois moiennes vertus] .iii. vers communication humaine en paroles et en faiz. » (Éthiques. Texte, p. 272). On constate, ici encore, l'insistance de la part d'Oresme sur l'alliance entre le discours et l'acte.

31. Dans le texte même d'Aristote, opposé à la glose, dans une phrase située après le passage cité, ce sont expressément les lois et les coutumes qui sont en relation avec le problème de l'immigration: "Car ce que aucuns viennent en la cité qui sunt nourris en lays et coustumes estranges, aucuns dient que ce est une chose non conferent ou nuisible a bonne legislation.» (Politiques. Texte, p. 295b) 
32. «Et ce n'est pas a entendre quant est de aler en mer pechier les poissons; mes pour aler de païz en autre par mer. Apres il met les nuisemens ou malz que aucuns disoient venir de ce.» (Politiques. Glose, p. 295b).

33. Le groupe de recherche du Dictionnaire du Moyen Français, dirigé par Robert Martin et faisant partie de l'Institut national de la Langue Française (CNRS), a fait saisir et mettre à la disposition des chercheurs une base de données textuelles relevant du moyen français.

34. Le concept de communauté prend une importance toute nouvelle au XIV ${ }^{\mathrm{e}}$ siècle. $\mathrm{Cf}$. sur ce point, B. Guénée, L'Occident aux XIV et XV siècles : les États, Paris, P.U.F., p. 152-153 ; en particulier, p. 153 : «Dieu, disent les théologiens, a confié l'autorité au peuple pour qu'il la transmette à un prince."

35. Tobler-Lommatzsch, II, 648.

36. Dans le même texte, on trouve la collocation monstre/insensible: «Et tel est comme un monstre et insensible.» (Éthiques. Commentaire, p. 225). Cette phrase se situe dans le contexte suivant appartenant à la même glose : "Mais deux autres manieres peuent estre : une est se aucun ne se delicte en telz choses pour deffaute de nature et pour la malice de sa complexion.» Les deux mêmes causes que celles qui ont été invoquées pour le passage réunissant agreste et insensible sont mentionnées ici : deffaute de nature et malice de sa complexion; ce dernier syntagme peut, semble-t-il, se rapprocher de fole acoustumance; en effet, la complexion (au sens de «tempérament ») est susceptible d'être influencée par l'éducation et l'environnement culturel. Il arrive chez Oresme que complexion soit associé à meurs des gens : «Car selon diversité des regions, des complexions, des inclinations et des meurs des gens, il convient que leur droiz positifs et leur gouvernemens soient differens. » (Politiques, p. 291b).

37. Ici, comme souvent, dans la période du moyen français, les deux termes sont équivalents.

38. Voir, sur ce point, B. Guénée, L'Occident aux XIVe et XV siècles. Les États, op. cit., p. 152 : « Le roi est donc sous la Loi, ou plutôt sous les Lois, et il doit les respecter toutes, la Loi naturelle comme la Loi divine, et aussi la Loi positive de son royaume où sont consignés les coutumes et les privilèges de son peuple. »

39. Oresme, dans ses Politiques, insiste longuement sur la question (notamment dans le livre V, chap. 11, p. 211).

40. Quelques phrases plus haut, la notion contraire faisait son apparition, mais pour être aussitôt limitée dans sa portée : "Car se aucunes fois les ydiotes ou ignorans participoient en bien eslire vers aucunes ouvres ou vers aucuns ars, toutesvoies il ne eslisent pas miex que ceulz qui sunt sachans. "

41. D’après les nombreux sondages effectués dans les diverses œuvres ne figurant pas dans la Base DMF et d'après le dépouillement exhaustif présenté par cette même Base pour le Livre de la mutacion, Charles V, le Livre du Chemin de long estude, le Livre de la paix.

42. Un cas semblable se retrouve dans les Politiques: «Comment les murs de la cité doivent estre faiz telement que en temps de paes et en temps de guerre il soient au parement de la cité et a la seurté de elle. » (Politiques. Glose., p. 360a). Ou encore, dans le Livre du ciel et du monde (p. 574) : « Et quartement: de ce que la terre est ronde comme dit est, il s'ensuit que se un honme povoit aler d'une cité a une autre sans monter et sans avaler, l'en puet ymaginer une autre voie plus brieve qui est partie en devalant et partie en montant. »

43. Voir notre article «Elévation, gloire et renommée dans quelques œuvres de Christine de Pizan ", Sur le chemin de longue étude... Actes du colloque d'Orléans juillet 1995, Textes réunis par B. Ribémont, Paris, Champion, 1998, pp. 45-64.

44. Cf. dans la citation précédente le mot maistre.

45. Quelquefois, c'est le terme nobilité, variante de noblece, qui apparaît aux côtés de cité :

Ou temps jadis lui plot mander [v. 4246]

Par son prophete en la cité,

Qui fu de grant nobilité, 
Ninive par nom appellee,

Qui riche fu et grant et lee [...] (Mutacion, t. I, p. 152).

46. Cette unité politique était, dans l'antiquité latine, assurée par le terme de civitas ; c'était le cas, tout particulièrement, du Bellum Gallicum de César.

47. Dans l'histoire de la langue française.

48. $\mathrm{Cf}$. la conception de la cité dans la période mérovingienne et carolingienne.

49. Élément de la topique médiévale, emprunté au De Senectute de Cicéron; voir plus particulièrement le chapitre XI (éd. W. A. Falconer, Harvard, 1964), pp. 42 sq. (The Loeb Classical Library, 45).

50. Christine ne se montre pas toujours tendre à l'égard du peuple. Voir, sur ce point, G. Mombello, «Quelques aspects de la pensée politique de Christine de Pizan d'après ses œuvres publiées ", Culture et politique en France à l'époque de l'Humanisme et de la Renaissance, Études réunies et présentées par Fr. Simone, Torino, Accademia delle scienze, 1974, p. 137.

51. C'est en particulier la répétition d'extrange/estrange qui conduit à proposer la valeur en question.

52. Implicitement, ici, Christine donne une leçon de politique pratique aux princes et à "son " prince. C'est dans cette optique que le terme désignant la sagesse pratique du chef d'État peut trouver sa place, à savoir la prudence; savoir discerner le mal et le bien pour soi et le pays que l'on gouverne, voilà sans doute une exigence essentielle dans un genre tel que celui des Miroirs des princes. Dans le Livre de la mutacion de Fortune, Christine ébauche l'analyse du concept de prudence tout en le replaçant, de façon laudative, dans le champ d'activité de Charles V :

Son filz Charles, roy quint du nom, [v. 23535]

En son jeune aage n'ot senon

Meschief, et tant divers assault,

Mais du mal el bien fist le saut,

Par son tres grant sens et prudence,

Car tant hot noble providence

Et avis que ses ennemis,

Qui environ luy furent mis,

De partout son regne chaça,

Et, moult s'accrut, tant pourchaça. (Mutacion, t. IV, p. 76).

53. [...] mais se aucuns vouloient dire que le contraire appaire par ce que plusieurs citéz en Ytalie et autre se gouvernent par les menus [...], je respons que voirement font, mais que de nulle aye ouy parler que bien par telz soit gouvernée, ne longuement à paix, je dis que non. (Paix, p. 132).

54. On admet que Le Livre du corps de policie a été achevé en 1407, Le Livre de la paix en 1413 ; cf. Ch. C. Willard, Christine de Pizan. Her life and works, New York, Persea Books, 1984, p. 177 et 190.

55. On peut au moins préciser qu'Oresme, à propos de son Livre du ciel et du monde, est mentionné dans Charles V, t. II, pp. 43-44.

56. On aurait pu, dans le même champ de recherche, étendre l'enquête à d'autres vocables. Nous avons retenu les plus abstraits dans l'ordre politique et social.

57. Mais, chez Oresme, les notions quelque peu techniques sont présentées dans une perspective pratique, de façon que le lecteur qui ignore les théories d'Aristote puisse parfaitement comprendre le discours d'Oresme. Cf. sur ce point K. L. Forhan, «Reading backwards: Aristotelianism ", Au champ des escriptures. III colloque international sur Christine de Pizan, Lausanne, 18-22 juillet 1998, Étudesréunies par E. Hicks, Paris, p. 381.

58. De ce point de vue, il est intéressant de noter qu'Oresme, en parlant de la puissance du roy, affirme: "Mes sa puissance doit estre moindre que celle de la multitude " (Politiques. Texte, p. 157a). La même idée est exprimée plus loin dans le même ouvrage : «[...] la puissance du roy doit estre moienne telement que elle soit plus grande que la puissance de chescun singulier et de pluseurs. Mes elle doit estre mendre que celle de toute la multitude ou de la plus vaillant partie. » 
(Politiques. Glose, p. 274b). Cf. sur cette question, S. M. Babbitt, op. cit., p. 108). On sait que Charles $\mathrm{V}$ se résigne à réaliser certaines réformes qu'avaient réclamées les états généraux, tout en sauvegardant l'essentiel du pouvoir royal.

59. Cf. S. M. Babbitt, op. cit., p. 75.

60. Christine est constamment préoccupée, et ce souvent affectivement, aussi bien de la gloire du roi, Charles V, que du bien-être du peuple; elle prend part aux situations les plus tragiques qui frappent l'un et l'autre.

61. Dans les gloses, à plusieurs reprises, Oresme évoque les soulèvements populaires de son époque, par lesquels la policie et la cité sont mises en cause.

62. C'est ainsi que dans les Éthiques, on peut lire (p. 435) : «Si come il advenoit en la policie de l'Église, que ceulz qui ont la souveraineté distribuassent les dignités et les biens as indignes, et que ilz en preïssent pou euls trop largement et feïssent que la souveraineté demouast a un lignage ou païs particulier [...]. »

63. Politiques. Glose, p. 160a : «Et ainsi, par aventure, pour ce que pluseurs par adulation ou par ignorance ont persudé as Sains Peres que il usoient de plenitude de posté et de reservations merveilles et de nouvelles exactions et de donner expectations qui semblent de prime face contraires as canons anciens. » Voir S. M. Babbitt, op. cit., pp. 111 sq.

\section{AUTEUR}

\section{CHARLES BRUCKER}

Université de Nancy-II 